\title{
Critical care management of COVID-19 infection in previously vaccinated patients - a case series
}

\author{
Anwar ul Huda, FRCA, FFICM, FCPS ${ }^{1}$, Amjad Mehboob, FCPS ${ }^{2}$ \\ Abdul Saboor Memon, FCPS $\triangle$
}

\section{Author affiliation:}

1. Consultant Anesthetist, Security Forces Hospital, PO Box: 3643, Salaheddine Road, Al Malaz District, Riyadh, Kingdom of Saudi Arabia

2. Senior Registrar Anesthesia, Security Forces Hospital, PO Box: 3643, Salaheddine Road, Al Malaz District, Riyadh, Kingdom of Saudi Arabia

Correspondence: Dr. Anwar ul Huda, FRCA, FFICM, FCPS; Email: hudaanwar90@yahoo.com

\section{Abstract}

Vaccination against COVID-19 has made significant contribution in reducing the severity of the disease and the mortality worldwide. Although most of the vaccinations have been reported to be safe and without serious side effects other than mild to moderate pain at the vaccination site, occasional reports of developing overt viral disease after the vaccination have emerged. We report a case series of four cases, who developed symptomatic COVID-19 following vaccination. We describe the clinical features and intensive care management of these patients.

Key words: COVID-19 infection; Vaccine; Critical care management; High flow nasal oxygen

Citation: Huda AU, Mehboob A, Memon AS. Critical care management of COVID-19 infection in previously vaccinated patients - a case series. Anaesth. pain intensive care 2021;25(6):793-797; DOI: 10.35975/apic.v25i6.1711

Received: September 08, 2021, Reviewed: September 19, 2021, Accepted: October 05, 2021

\section{Introduction}

The world is currently facing one of the worst pandemics in the history of mankind, caused by severe acute respiratory syndrome corona virus 2 (SARS-CoV-2). ${ }^{1}$ The presentation of this disease ranges from mild symptoms, including dry cough, fever to severe pneumonia leading to respiratory failure, sepsis and ultimately death. ${ }^{2}$ Efforts to control the spread of the virus with personal protective actions (e.g., reduced interpersonal contact, physical distancing, hand washing and mask wearing), or national level initiatives (e.g., travel restrictions, closure of schools and lockdowns) have suppressed transmission to some extent but these fail to alter the fundamentals of the pandemic. ${ }^{3}$

Vaccination on a national and global scale provides an opportunity to control this pandemic. ${ }^{4}$ Menni et al. reported an infection risk reduction at 21-44 days postvaccination in all vaccinated users compared with controls (RR was between -60 to $-69 \%$ for two types of vaccines). ${ }^{5}$ Another study concluded that a symptomatic
COVID-19 rate was 5 per 10,000 person-days in unvaccinated healthcare personnel (HCP); the disease rates were 2.8 and 1.2 per 10,000 person-days on days 1 14 and days 15-28 after first dose of vaccine, respectively. ${ }^{6}$ Eric et al. showed that two doses of mRNA vaccines were found to be highly effective against COVID-19 infections and hospitalizations, severe disease and death. ${ }^{7}$

We present this case series of four patients, who developed COVID-19 pneumonia after receiving vaccination. We describe their clinical characteristics and critical care management.

\section{Case Report 1}

A 63 years old male, a known case of diabetes type-2, dyslipidemia and hypertension, was admitted to emergency room with complaints of cough, fever, generalized fatigability for the last 7 days. He also had breathing difficulty for one day. His symptoms started 7 days after taking first dose of the Pfizer-BioNTech 
COVID-19 vaccine and his COVID-19 polymerase chain reaction (PCR) test was positive. The patient was admitted to the COVID ward and was started on minimal oxygen support along with IV ceftriaxone, azithromycin, dexamethasone, enoxaparin and favipiravir. On day 6 of admission, patient's $\mathrm{SpO}_{2}$ dropped to $86 \%$, which was managed with face mask at $6 \mathrm{~L} / \mathrm{min}_{2}$. On the next day (D7), due to desaturation the $\mathrm{O}_{2}$ requirement was escalated to high flow nasal cannula (HFNC) and given $60 \mathrm{~L} / \mathrm{min}$ with $\mathrm{FiO}_{2} 95 \%$. Chest X-ray showed worsening of bilateral infiltrates. Antibiotic was upgraded to inj. piperacillin/tazobactam and the first dose of inj. tocilizumab was given. On day 10 of admission, the patient had to be admitted to intensive care unit (ICU) due to worsening hypoxia, tachypnea and high $\mathrm{O}_{2}$ requirement with $\mathrm{HFNC}$ at $60 \mathrm{~L} / \mathrm{min}$ plus nonrebreathing mask (NRM) $15 \mathrm{~L} / \mathrm{min}$. In ICU, all routine care, along with piperacillin/tazobactam, enoxaparin, favipiravir, aspirin, incentive spirometry and chest physiotherapy, was provided. His serum LDH, ferritin and CRP levels reached at maximum on day 4 after which these started dropping as shown in Figure 1 and 2. His WBC stayed within normal limits for the first 10 days, though it increased to the maximum level at day 12 before dropping again as shown in Figure 2. His oxygen requirement remained at very high level for 4-5 days. Patient stayed in ICU for 9 days, during which $\mathrm{O}_{2}$ requirement was weaned to nasal cannula at $3 \mathrm{~L} / \mathrm{min}$. He was shifted to the ward and then discharged from the hospital on day 22 of admission on room air in stable condition.

\section{Case Report 2}

A 53-year-old male, a known case of diabetes mellitus, hypertension and post renal transplant, on immunosuppression therapy, was admitted. His COVID swab became positive two months after completing Pfizer COVID-19 vaccination. He presented to Emergency Department with complaints of fever, shortness of breath, cough, diarrhea and decreased oral intake for 6 days. His $\mathrm{SpO}_{2}$ was $90-91 \%$ on $3 \mathrm{~L} / \mathrm{min}$ oxygen via nasal cannula. He was admitted in the ward and commenced on intravenous azithromycin, ceftriaxone, favipiravir, prophylactic heparin and dexamethasone. Immunosuppressant tarcolimus along with carvedilol were continued. His oral prednisolone was held on the advice of nephrologist. On day 2 of admission, his oxygen requirement increased and he was placed on HFNC at $60 \mathrm{~L} / \mathrm{min}$ with a non-rebreather mask (NRM) and was transferred to ICU. His antimicrobial coverage was changed from ceftriaxone to piperacillin/tazobactam. His serum LDH, ferritin and CRP levels reached at maximum value on day 3; after that it started decreasing as shown in Figure 1 and 2. His WBC count reached maximum on day 10 and then it decreased as shown in Figure 2. His liver enzymes remained normal during his ICU stay. The creatinine level was slightly raised during 1st week which normalized later on. On day 5 of the hospital admission, his anticoagulation was upgraded to enoxaparin $40 \mathrm{mg}$ twice daily due to rising D-dimer. On day 9, tocilizumab $700 \mathrm{mg}$ was administered and the antibiotic was changed to meropenem. After 12 days, his oxygen requirement started falling. On day 14 , he was able to maintain oxygenation on $15 \mathrm{~L} / \mathrm{min}$ of oxygen via NRM. On day 20 , he was placed on $6 \mathrm{~L} / \mathrm{min}$ of $\mathrm{O}_{2}$ through a nasal cannula. On day 22, he was shifted to the ward on nasal cannula at $2 \mathrm{~L} / \mathrm{min}$. There was no growth detected in various culture examinations. He remained hemodynamically stable throughout his hospital stay. On day 27 , he was discharged home without oxygen supplementation.

\section{Case Report 3}

A 68 years old lady was a known case of diabetes mellitus, hypertension, dyslipidemia, atrial fibrillation, fatty liver, hypothyroidism, chronic kidney disease stage 3-4 and obesity. She underwent transcatheter aortic valve implantation (TAVI) and permanent pace maker insertion one year back. Her COVID-19 swab became positive 2 weeks after receiving first dose of AstraZeneca-Oxford vaccine. She reported to the emergency department with the complaints of fever, cough, shortness of breath and nausea for one week. Her $\mathrm{SpO}_{2}$ was 93-94\% on room air and she was vitally stable. She was admitted to the ward and was started on intravenous ceftriaxone, azithromycin, favipiravir and dexamethasone along with her usual home medications. On day 3, her oxygenation got worsened and she was started on $\mathrm{HFNC}$ at $50 \mathrm{~L} / \mathrm{min}$ with $\mathrm{FiO}_{2}$ kept at $70 \%$. She was transferred to the ICU, and the antibiotic cover was changed to meropenem and routine ICU care was instituted along with subcutaneous heparin 5000 IU twice daily. Intravenous tocilizumab $800 \mathrm{mg}$ was administered as well. Her serum LDH, ferritin and CRP levels reached at maximum at 5-7 days after admission as shown in Figures 1 and 2. Her WBC count remained within normal limits during her stay at ICU as shown in Figure 2.

Liver enzymes were mildly deranged during the first week but improved later on. Her creatinine level also rose above normal for the first 7 days, after which it decreased to her baseline level. We tried to keep her in negative fluid balance during her ICU stay. On day 5, she was able to maintain oxygen saturation at $95-98 \%$ with HFNC 50 $\mathrm{L} / \mathrm{min}$ and $\mathrm{FiO}_{2}$ of $60 \%$. On day 8 , she was placed on 6 $\mathrm{L} / \mathrm{min}$ of oxygen through a face mask. On day 10 , she was transferred to the ward on nasal cannula at $2 \mathrm{~L} / \mathrm{min}$. On day 11, she was discharged home fully recovered without supplemental oxygenation. 


\section{Case Report 4}

A 41 years old male, with no previously known medical comorbids, was admitted to the ward with complaints of cough and shortness of breath. He had received PfizerBioNTech COVID-19 vaccine 10 days before his symptoms. On admission, he was started on oxygen via facemask, and intravenous favipravir, azithromycin, dexamethasone, piperacillin/tazobactam (Tazocin $\left.{ }^{\circledR}\right)$ and enoxaparin. Tocilizumab was also administered intravenously. His oxygen requirement continued to increase and he was started on HFNC along with NRM and shifted to ICU. On ICU admission, he was in mild respiratory distress and his vitals included heart rate 45 beats/min, blood pressure 110/60 $\mathrm{mmHg}$ and $\mathrm{SpO}_{2} 87 \%$. His arterial blood gases showed PO2 $60 \mathrm{mmHg}$ and $\mathrm{PCO}_{2}$ $33 \mathrm{mmHg}$. Arterial line was inserted in the left radial artery. Chest X-ray showed bilateral infiltrates. He was encouraged for self proning and incentive spirometry.

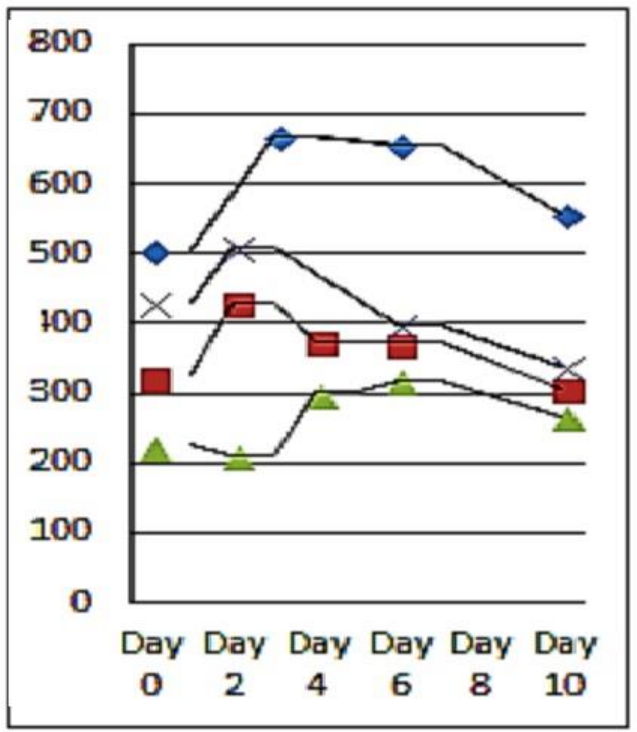

LDH levels (U/L)

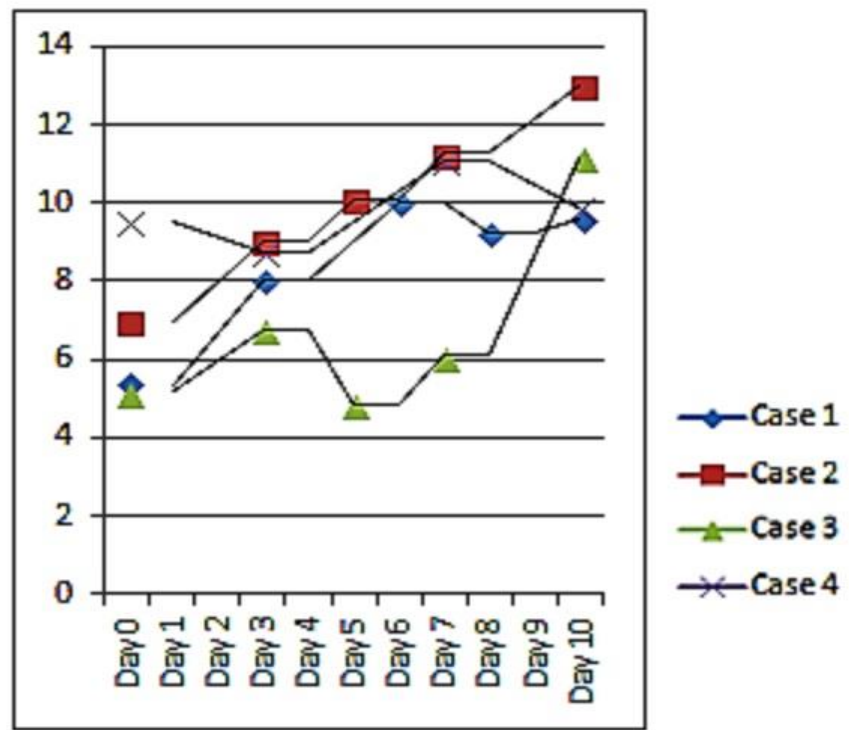

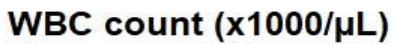

Figure 2: Line graphs showing blood levels of C-reactive protein and ferritin in 4 patients

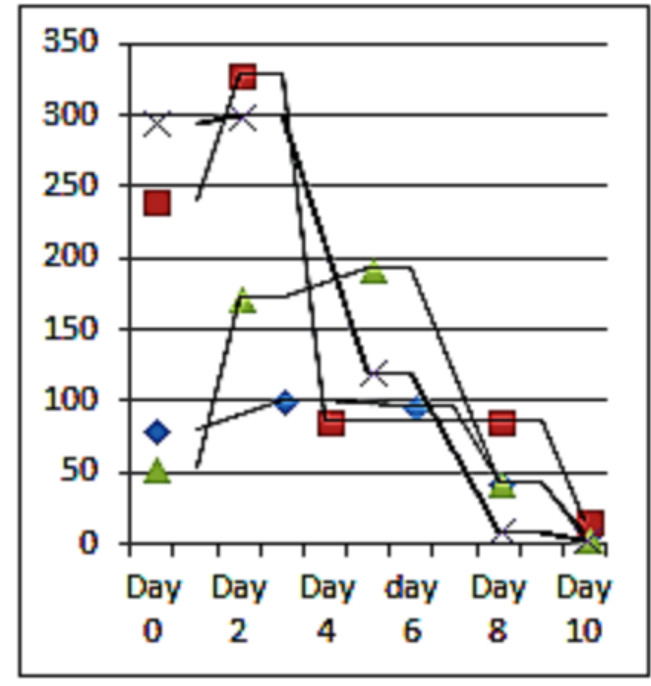

CRP levels (mg/dL)

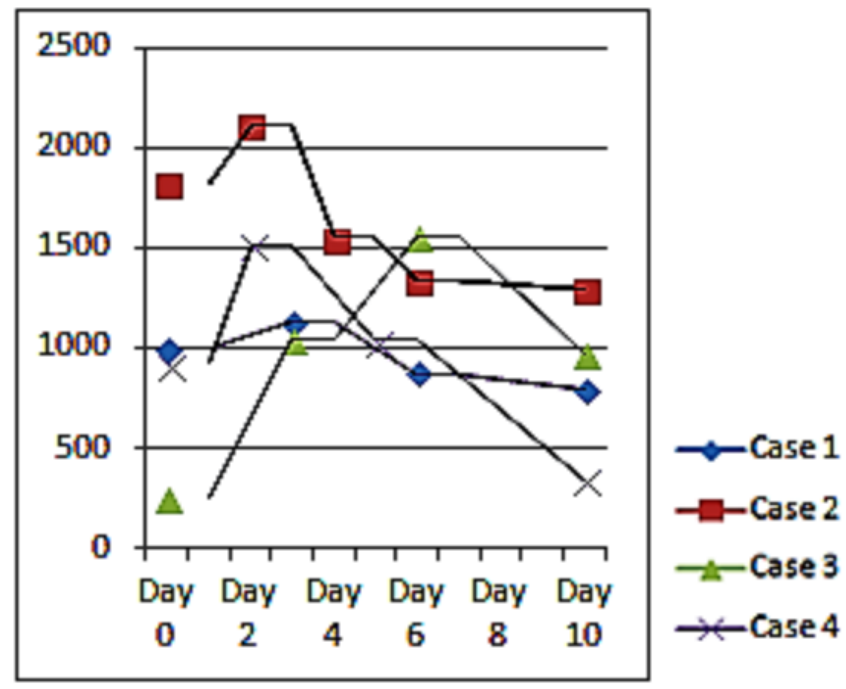

Ferritin levels $(\mu \mathrm{g} / \mathrm{L})$

Figure 1: Line graphs showing blood levels of LDH and WBC count in 4 patients 
His serum LDH, ferritin and CRP reached maximum values on day 2 of ward admission as shown in Figure 1 and 2. The WBC count stayed within normal limits during his ICU course as shown in Figure 2. On day 4 of admission, gram negative rods were demonstrated in his arterial line, so it was replaced. His liver enzymes deranged on day 4, which later improved. His oxygen requirement started to come down on day 5 . On day 7 , he was shifted to ward on face mask at $5 \mathrm{~L} / \mathrm{min}$ of $\mathrm{O}_{2}$.

\section{Discussion}

In this case series, we describe four patients who were admitted in our ICU with symptomatic COVID-19 infection despite being vaccinated against the virus. All patients were tested positive in nasopharyngeal swab for COVID-19 PCR prior to admission to the hospital. The most common presentation in our case series was cough and shortness of breath (in all of the four subjects). Other symptoms were fever (in three subjects), diarrhea, nausea, generalized fatigability and decrease oral intake (each in one of the subjects). Scientific literature shows that the most common symptoms in symptomatic COVID-19 patients were cough (89.4\%), fever (85.6\%), and sore throat (81.6\%).8 COVID-19 patients mostly present with fever, dry cough, and fatigue. A number of less frequent symptoms include anosmia (loss of smell), hypogeusia (loss of taste), diarrhea, and headache etc. In more severe cases, patients may have chest pain, dyspnea, chest pain, and myalgia or even loss of speech or movement. ${ }^{9.10}$ Almalki et al. demonstrated that fever, dry cough, dyspnea, sore throat, diarrhea, fatigue, and vomiting were the most commonly reported symptoms, whereas runny nose, chest pain, abdominal pain, myalgia, and nausea were relatively rare. ${ }^{11}$

Three patients in our case series developed symptomatic COVID-19 infection within 14 days of getting first dose of vaccination. Two out of them received Pfizer vaccine and one received Astra-Zeneca. One patient completed Pfizer vaccination 2 months prior to symptomatic COVID-19 infection. In our case series, two patients received one dose of Pfizer vaccine less than 14 days prior to demonstrating severe symptomatic COVID-19 infection that was in the time window where immune response is not optimum. The only patient who completed 2 doses of Pfizer vaccine more than 14 days (two months) prior to exhibiting severe symptomatic COVID-19 infection was on immunosuppressant therapy due to renal transplantation. It has been demonstrated that there is significant reduction in immune response to Pfizer vaccine in renal transplant recipients. ${ }^{12}$

Abnormal laboratory findings in mild and moderate severity patients were shown to be; CRP -11.1 vs $28.6 \mathrm{mg} / \mathrm{L}$, D-dimer - 0.57 vs $0.69 \mathrm{mg} / \mathrm{L}$ and ferritin 201.6 vs $445.2 \mathrm{ng} / \mathrm{ml}$. Neutrophil count, lymphocyte count, alanine aminotransferase, aspartate aminotransferase, CRP, D-dimer, and ferritin were even higher in the COVID-19 moderate severity patients. ${ }^{13}$ In our case series, all our patients had raised levels of CRP, ferritin and LDH which peaked at day 3-5 after hospital admission.

All patients received tocilizumab according to the local protocol, except for the renal transplant recipient patient who was initially not given tocilizumab in view of his immunosuppression; however, it was administered later during his ICU stay. Regarding anticoagulation, three patients were given enoxaparin therapeutic doses based on their D-dimer values. One patient was given heparin due to renal derangement. We routinely administered therapeutic enoxaparin for patients with D-dimer more than one $\mu \mathrm{g} / \mathrm{ml}$. All patients received dexamethasone, favipiravir, azithromycin and ceftriaxone. Antibiotics were later upgraded based on clinical findings and culture results.

In patients with chronic conditions, significant differences have been reported in the severity of dyspnea. It is presumed that individuals with chronic conditions are more prone to severe infection; therefore, it has been suggested that the initial onset of dyspnea may indicate a poor prognosis. ${ }^{14}$ Patients with risk factors and underlying health conditions, including diabetes mellitus, hypertension, coronary artery disease, COPD, cerebrovascular disease, and chronic renal disease, are at an elevated chance of severe illness or high COVID-19 mortality. ${ }^{9}$ In our case series, 3 out of 4 patients had significant comorbids. Despite significant medical comorbids, all of them had excellent prognosis in our case series.

There are various limitations in our study. It is a small case series, managed at a single center without long term outcome. In conclusion, vaccination is helpful in avoiding serious morbidity and mortality in COVID-19 patients.

\section{Financial support}

None declared by the authors. No external funding was involved in the preparation of this manuscript.

\section{Conflicts of interest}

None declared by the authors.

\section{Authors" contribution}

AUH: Concept, Literature search, Manuscript writing/editing

AM: Study design, Data analysis, Manuscript editing

ASM: Literature search, Data collection, Manuscript editing

All authors approved the final draft of the manuscript/ 


\section{References}

1. Lotfi M, Hamblin MR, Rezaei N. COVID-19: Transmission, prevention, and potential therapeutic opportunities. Clin Chim Acta. 2020 Sep;508:254-266. [PubMed] DOI: 10.1016/j.cca.2020.05.044

2. Zhu N, Zhang D, Wang W, Li X, Yang B, Song J, et al. China novel coronavirus investigating and research team. a novel coronavirus from patients with pneumonia in China, 2019. N Engl J Med. 2020 Feb 20;382(8):727-733. [PubMed] DOI: 10.1056/NEJMoa2001017

3. Cook TM, Roberts JV. Impact of vaccination by priority group on UK deaths, hospital admissions and intensive care admissions from COVID-19. Anaesthesia. 2021 May;76(5):608-616. [PubMed] DOI: 10.1111/anae.15442

4. Gong Y, McDonald S. How logical is the UK's vaccine priority ordering? COVID-19 Actuaries Response Group. 7 December 2020. Available from: https://www.covid-arg.com/post/vaccinepriorities (accessed 30/08/2021).

5. Menni C, Klaser K, May A, Polidori L, Capdevila J, Louca P, et al. Vaccine side-effects and SARS-CoV-2 infection after vaccination in users of the COVID Symptom Study app in the UK: a prospective observational study. Lancet Infect Dis. 2021 Jul;21(7):939-949. [PubMed] DOI: 10.1016/S14733099(21)00224-3

6. Amit S, Regev-Yochay G, Afek A, Kreiss Y, Leshem E. Early rate reductions of SARS-CoV-2 infection and COVID-19 in BNT162b2 vaccine recipients. Lancet. $2021 \mathrm{Mar}$ 6;397(10277):875-877. [PubMed] DOI: 10.1016/S0140$6736(21) 00448-7$

7. Haas EJ, Angulo FJ, McLaughlin JM, Anis E, Singer SR, Khan $F$, et al. Impact and effectiveness of mRNA BNT162b2 vaccine against SARS-CoV-2 infections and COVID-19 cases, hospitalisations, and deaths following a nationwide vaccination campaign in Israel: an observational study using national surveillance data. Lancet. 2021 May 15;397(10287):1819-1829. [PubMed] DOI: 10.1016/S0140-6736(21)00947-8

8. Alsofayan YM, Althunayyan SM, Khan AA, Hakawi AM, Assiri AM. Clinical characteristics of COVID-19 in Saudi Arabia: A national retrospective study. J Infect Public Health. 2020 Jul;13(7):920-925. [PubMed] DOI: 10.1016/j.jiph.2020.05.026

9. Guan WJ, Ni ZY, Hu Y, Liang WH, Ou CQ, He JX, et al. Clinical characteristics of coronavirus disease 2019 in China. N Engl J Med. 2020 Apr 30;382(18):1708-1720. [PubMed] DOI: 10.1056/NEJMoa2002032

10. Wang D, Hu B, Hu C, Zhu F, Liu X, Zhang J, et al. Clinical characteristics of 138 hospitalized patients with 2019 novel coronavirus-infected pneumonia in Wuhan, China. JAMA. 2020 Mar 17;323(11):1061-1069. [PubMed] DOI: 10.1001/jama.2020.1585

11. Almalki ZS, Khan MF, Almazrou S, Alanazi AS, lqbal MS, Alqahtani $A$, et al. Clinical characteristics and outcomes among covid-19 hospitalized patients with chronic conditions: a retrospective single-center study. J Multidiscip Healthc. 2020 Oct 6;13:1089-1097. [PubMed] DOI: 10.2147/JMDH.S273918

12. Korth J, Jahn M, Dorsch O, Anastasiou OE, Sorge-Hädicke B, Eisenberger $U$, et al. Impaired humoral response in renal transplant recipients to SARS-CoV-2 vaccination with BNT162b2 (Pfizer-BioNTech). Viruses. 2021 Apr 25;13(5):756. [PubMed] DOI: 10.3390/v13050756

13. Al Mutair A, Alhumaid S, Alhuqbani WN, Zaidi ARZ, Alkoraisi S, Al-Subaie MF, et al. Clinical, epidemiological, and laboratory characteristics of mild-to-moderate COVID-19 patients in Saudi Arabia: an observational cohort study. Eur J Med Res. 2020 Nov 25;25(1):61. [PubMed] DOI: 10.1186/s40001-020-00462-x

14. Zhang J, Wang X, Jia X, Li J, Hu K, Chen G, et al. Risk factors for disease severity, unimprovement, and mortality in COVID19 patients in Wuhan, China. Clin Microbiol Infect. 2020 Jun;26(6):767-772. [PubMed] DOI: 10.1016/j.cmi.2020.04.012 